\title{
Verbreitung, Typ und Alter der maximalen Vergletscherung der Südvogesen zwischen Bruyères und Epinal
}

\author{
GERD WENZENS *) \\ Pleistocene, glaciation, Mindel Glaciation, Riss Glaciation, Würm Glaciation, \\ ice-marginal features, sections, erratics, moraines, glaciofluvial sediments, paleosols, geomorphology \\ France, Vosges Mountains
}

\begin{abstract}
Kurzfassung: Die weiteste Ausdehnung der maximalen Vergletscherung zwischen Bruyères und Epinal ist äußerst umstritten. Während sie nach RAHM (1970: Karte 1) und HANTKE (1978: Karte 2) in diesem Raum in Form einer Kappenvereisung bis an den Fuß des Horstes von Epinal gereicht haben soll, gehen SERET (1980: Fig. 1) und FLAGEOLLET (1988: Abb. 7) von einer wesentlich geringeren Vergletscherung aus: Nach ihren Auffassungen stirnte der VologneGletscher nördlich von Granges-sur-Vologne (Abb. 1).

Verschiedene Geländebefunde und Berechnungen der Eismächtigkeit weisen demgegenüber auf eine insgesamt wesentlich größere Ausdehnung der maximalen Vergletscherung hin. Zwischen dem Moseldurchbruch und Bruyères stimmte die nördliche Grenze in etwa mit dem von RAHM und HANTKE angenommenen Verlauf überein. Im Gegensatz zu RaHM und HANTKE, die ein rißeiszeitliches Alter der Vergletscherung postulieren, stellt der Verfasser diesen Vorstoß aufgrund des Verwitterungsgrades der Deckschichten und der noch wenigen erhaltenen Erratika in die MindelKaltzeit.
\end{abstract}

[Range, Type and Age of the Maximum Glaciation in the Southern Vosges between Bruyères and Epinal]

Abstract: The widest range of the maximum glaciation between Bruyères and Epinal is very controversial. Following RAHM (1970: map 1) and HANTKE (1978: map 2) it was an ice cap, reaching just to the horst of Epinal. On the other hand SERET (1980: map 1) and FLAGEOLLET (1988: map 7) postulate a smaller range of the ice. From their point of view the Vologne glacier only advanced to the north of Granges-surVologne (see map 1).

A lot of facts resulting from field studies and calculations of ice thickness point to a much wider range of the maximum glaciation. Between the Mosel gap and Bruyères the northern limit is nearly identical with the one that RAHM and HANTKE mapped. In contrast to RAHM and HANTKE, who dates the

*) Anschrift des Autors: Prof. Dr. G. Wenzens, Geographisches Institut der Universität Düsseldorf, Universitätsstraße 1, D - 4000 Düsseldorf 1. maximum glaciation as rissian, the author postulates its mindel age because of the high grade of weathering of the cover and the rareness of erratic blocks.

[Extension, type et age de la glaciation maximale des Vosges sud entre Bruyères et Epinal]

Résumé: La plus grande extension de la glaciation maximale entre Bruyères et Epinal est discutée controversement. Suivant RAHm (1970: Fig. 1) et HaNTKe (1978: Fig. 2) elle s'élargit en forme d'une calotte glaciaire jusqu'au pied de l'horst d'Epinal. En opposition, Seret (1980: Fig. 1) et FLAGEOLLET (1988: Fig. 7) supposent une extension beaucoup moins large de la glace: selon leur conception le glacier de la Vologne avançait jusqu'au nord de Granges-surVologne (voir carte 1).

Divers résultats d'investigations du terrain et de calculations concernant l'épaisseur de la glace indiquent une extension beaucoup plus vaste de la glaciation maximale. Entre la cluse de la Moselle et Bruyères la limite nord coincide avec celle que RAHM et HantKe avaient marquée. RaHm et HANTKE donnent un age Rissien à la glaciation maximale. L'auteur au contraire, se référant au degré d'altération des couches de couverture et à la quantité restreinte des blocs erratiques, date cette avance glaciaire comme Mindelien.

\section{Einleitung}

\subsection{Das Untersuchungsgebiet}

Das Untersuchungsgebiet (Abb. 1) nimmt den nordwestlichen Teil der Südvogesen ein, der geologisch durch den stark zerlappten Übergang vom Grundzum mesozoischen Deckgebirge gekennzeichnet ist. Orographisch erfolgt ein allmählicher Abfall der ca. 600-700 $\mathrm{m}$ hohen Kristallinrücken zu den meist 400-600 m hohen Buntsandsteinflächen. Ausnahmen bilden die aus Buntsandstein aufgebauten Inselberge Spiemont (809 m ü. M.) und l'Ormont (827 m ü. M.), die ihre Umgebung um $100 \mathrm{~m}$ überragen. 


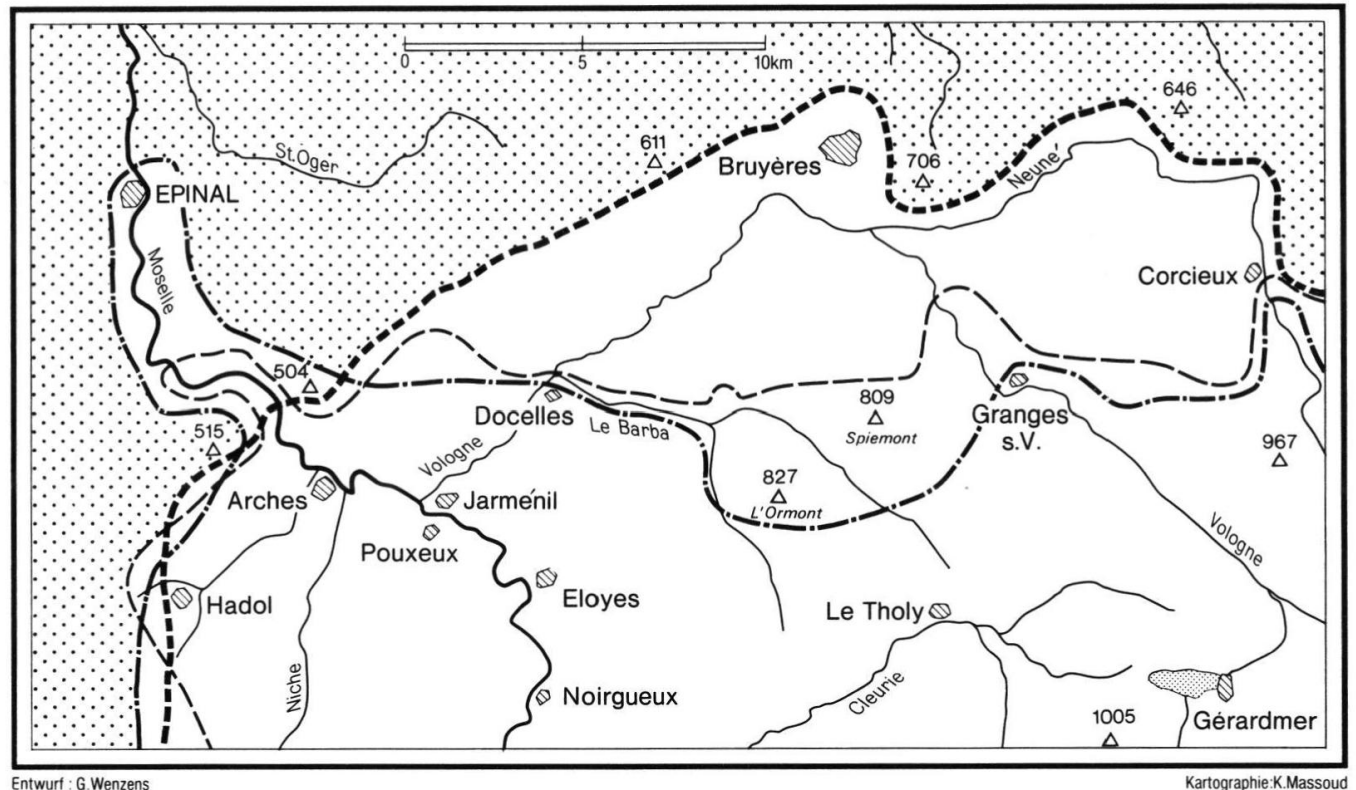

- - RAHM (1970) u. HANTKE (1978) —— FLAGEOLLET (1984) - - SERET (1980)

Abb. 1: Grenzen der maximalen Vergletscherung nach

Fig. 1: Limites de la glaciation maximale suivant

Rahm (1970) und HantKe (1978), Seret (1980), Flageollet (1984).

Im $\mathrm{N}$ erheben sich noch einmal die bewaldeten Rücken des SW-NE-streichenden Horstes von Epinal auf stellenweise über $500 \mathrm{~m}$ (nordwestlich von Arches $515 \mathrm{~m}$ ü. M., westlich von Bruyères $611 \mathrm{~m}$ ü. M.). Die Mosel durchbricht diesen Höhenzug nördlich Arches mit einem bis $340 \mathrm{~m}$ ü. M. eingetieften Engtal. Wichtigster Zufluß ist die Vologne, die sich zwischen Bruyères und ihrer Mündung in einem Abstand von 2,5 bis $4 \mathrm{~km}$ parallel zum Horst von Epinal eingeschnitten hat. Angelegt wurde dieser Talabschnitt allerdings von der Neuné, denn bis zum Ende der Würmeiszeit bildete das Cleurietal den Unterlauf der Vologne (WENZENS 1987: 80).

\subsection{Stand der Forschung zur Problematik der Vergletscherung im Untersuchungsgebiet}

In den letzten Jahren haben HANTKE (1978: Karte 2), SERET (1980: Fig. 1) und Flageollet (1984: Fig. 1, 1988: Abb. 7) Karten zur Vergletscherung der Südvogesen vorgelegt. HANTKE gliedert zwei Hauptvereisungen aus, wobei die Rißkaltzeit als die älteste Vergletscherung in Form einer Kappenvereisung das gesamte Untersuchungsgebiet zwischen Bruyères und dem Moseldurchbruch des Horstes von Epinal einnimmt (Abb. 1). Die Darstellungen von SERET und
FLAGEOLLET unterscheiden sich von dieser Interpretation sowohl in bezug auf die Zahl als auch auf die maximale Ausdehnung der Vereisungen. Sie gehen von insgesamt drei Hauptvergletscherungen aus und differenzieren zwischen einer älteren Plateauvergletscherung und zwei Talvergletscherungen. Während jedoch nach SERET (Abb. 2) die beiden Talvergletscherungen nur geringfügige Abweichungen bezüglich der jeweiligen Ausdehnung aufweisen und deutlich kleinere Flächen einnehmen, reichen bei FLAGEOLLET (Abb. 3) die Talgletscher der mittleren Vereisung verschiedentlich fast bis an die äußere Grenze der Plateauvergletscherung heran. Eine Ausnahme bildet bei beiden Autoren das Moseltal, in dem der Abstand zwischen ältester Plateau- und mittlerer Talvergletscherung $10-12 \mathrm{~km}$ beträgt.

Auf der geologischen Karte Bruyères wird im wesentlichen die von FLAGEOLLET vertretene Auffassung übernommen und ebenfalls zwischen den beiden Talvergletscherungen Würm und Riß sowie der mindelzeitlichen Kappenvereisung unterschieden. Auch SERET (1966: Fig. 72) hatte zunächst die drei Vergletscherungsphasen mit Würm, Riß und Mindel parallelisiert, jedoch später (1980: Fig. 1) aufgrund der Auswertung des Pollenprofils von Grande Pile durch WOILLARD (1978) nur noch zwischen einer älteren, 
mittleren und jüngeren Vereisung differenziert, wobei er dann (1985: 19) eine Gleichsetzung der älteren mit der Saale-, der mittleren mit der älteren Weichsel- und der jüngeren mit der jüngeren Weichselvereisung für wahrscheinlich hielt.

$\mathrm{Zu}$ diesen stratigraphischen Fragen hat zuletzt FLAGEOLLET (1988: $31 \mathrm{f}$.) Stellung genommen und überzeugende Gründe für die Beibehaltung der alpinen Nomenklatur angeführt.

\subsection{Methoden und Ziele}

Im folgenden soll nun versucht werden, den maximalen Eisvorstoß zwischen Epinal und Bruyères zu erfassen, d. h. den Raum zwischen der ältesten und der mittleren Vereisung anhand verschiedener Kriterien zu analysieren.

Die unterschiedlichen Abgrenzungen der mittleren Vergletscherung in den Karten von SERET (Abb. 2) und FlageOllet (Abb. 3) deuten die Schwierigkeiten dieses Unterfangens bereits an. Anhand von Endmoränenresten im Mosel- und Barbatal konnten die weitesten Vorstöße dieser Talgletscher während der mittleren Vereisung festgestellt werden. Jenseits dieser Vorkommen treten kaum noch eindeutig ansprechbare Glazialformen auf. Das Ausmaß der ältesten Vergletscherung kann daher vor allem durch den Nachweis von Erratika rekonstruiert werden. Hierbei handelt es sich i. w. um paläozoische Geschiebe (diverse Granitvarietäten, Gneise usw.), die dem Buntsandstein aufliegen. Während solche Findlinge
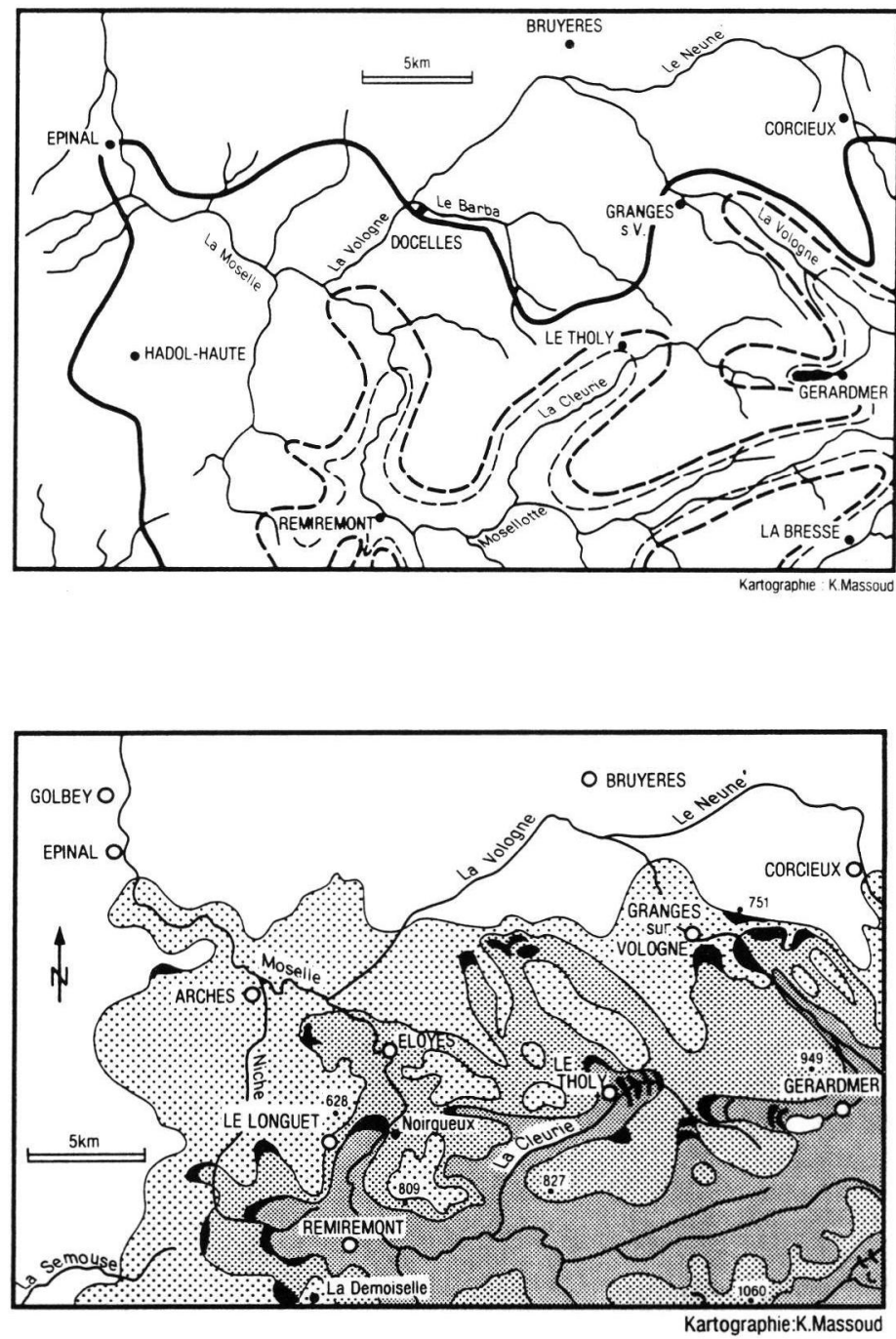

Abb. 2:

Grenzen der Vereisungen nach SERET (1980: Fig. 1)

Fig. 2:

Limites des glaciations suivant SERET (1980: Fig. 1)

\begin{tabular}{llll} 
& 1966 & 1980 & 1985 \\
\hdashline & Mindel & Ältere & Saale \\
$\ldots \ldots-$ Riß & Mittlere & Ältere Weichsel \\
$\ldots \ldots$ & . . . . Würm & Jüngere & Jüngere Weichsel
\end{tabular}

Abb. 3:

Grenzen der Vereisungen nach Flageollet (1984: Fig. 1)

Fig. 3:

Limites des glaciations suivant Flageollet (1984: Fig. 1)

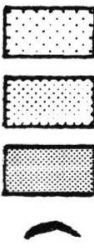

ältere Vereisung(en)/ glaciation(s) ancienne(s)

Riß

Würm

Endmoräne / moraine frontale 
im Moselbereich häufig eine Längsachse von über einem Meter aufweisen, sind die Kristallingeschiebe im östlichen Untersuchungsgebiet im allgemeinen wesentlich kleiner. Die Funde beschränken sich allerdings keineswegs nur auf einzelne Geschiebe an der Oberfläche, sondern treten auch in Anschnitten von Baugruben und im Wurzelwerk umgestürzter Bäume auf, so daß zumindest in den beiden letzten Fällen anthropogene Einflüsse weitgehend ausgeschlossen werden können. Unabhängig davon ist sich der Verfasser bewußt, daß das Auftreten einzelner Geschiebe recht unterschiedliche Interpretationen zuläßt (vgl. SCHREINER 1986).

Es wurde daher versucht, mit Hilfe hochgelegener Erratika (Großblöcke über $0,75 \mathrm{~m}$ Längsachse) die Eismächtigkeit in benachbarten Tälern zu rekonstruieren und so eine Vorstellung von der maximalen Ausdehnung der Gletscher zu gewinnen.

Ein weiteres Ziel der Arbeit ist es, die Frage nach dem Alter der maximalen und mittleren Vergletscherung zu klären, d. h. Anhaltspunkte dafür zu finden, ob die älteste Vereisung der Mindel- und die mittlere der Riß-Kaltzeit zugeordnet werden kann. Dies soll zunächst für das Gebiet des Moseltales untersucht werden. Anschließend wird der Raum nördlich der Linie Docelles-Granges s. V. auf Belege für die Zahl der Vergletscherungen hin überprüft.

\section{Glaziale und fluvioglaziale Ablagerungen im Bereich des Moseltales}

\subsection{Alter und Lage des Vergletscherungsrandes der mittleren Vereisung}

Nach übereinstimmender Auffassung von SERET (Abb. 2) und FlageOllet (Abb. 3) stirnte der Moselgletscher während der mittleren Vereisung auf der Höhe von Jarménil und Pouxeux und hinterließ hier in 420-450 m Höhe Endmoränenbögen. Die korrelaten proglazialen Ablagerungen sind in der $1,5 \mathrm{~km}$ entfernten Kiesgrube Les Côtes auf der rechten Moselseite angeschnitten (Abb. 4). Die Kiesgrube befindet sich am Rande einer Verebnung, die von 397-408 m ü. M. nach $\mathrm{N}$ auf $385 \mathrm{~m}$ ü. M. abfällt.

Die insgesamt $20 \mathrm{~m}$ mächtigen glazifluvialen Sedimente lassen sich in drei Ablagerungszyklen gliedern, die sich durch die Intensität der Schichtung und Sortierung sowie durch die variable Dominanz von Grobund Feinmaterial unterscheiden und so die jeweilige Fließdynamik der Schmelzwässer widerspiegeln (vgl. VASKOU 1984: 100f.).

Das hangende Paket zeichnet sich durch den größeren Anteil von Grobblöcken sowie fehlende Schichtung

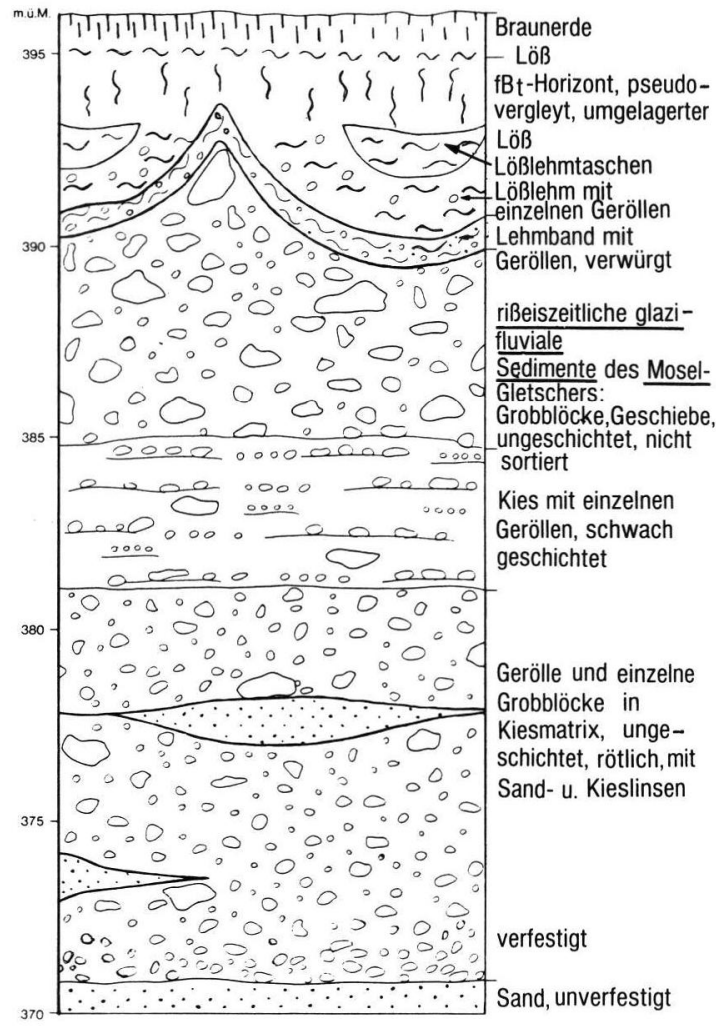

Abb. 4: Aufschluß Les Côtes (östlich Archettes): rißeiszeitliche glazifluviale Sedimente des Mosel-Gletschers und ihre Deckschichten.

Fig. 4: Coupe Les Côtes (à l'est d'Archettes): sédiments glaciofluviaux du glacier Rissien de la Moselle et leurs couches de couverture.

und Sortierung aus. Die oberen 5-8 $\mathrm{m}$ sind stark kryoturbat gestört, wobei die Schotter mit der aufliegenden Lößlehmdecke verwürgt sind. Der solifluidal umgelagerte Löß schließt mit einem nicht mehr verwürgten, ca. $60 \mathrm{~cm}$ mächtigen $B_{t}$-Horizont $a b$, der durch eine nachfolgende Pseudovergleyung überprägt worden ist. Überlagert wird dieser Komplex von einer weiteren ca. $50 \mathrm{~cm}$ mächtigen Lößdecke, auf der sich eine Braunerde entwickelt hat.

Die Intensität des $\mathrm{B}_{\mathrm{t}}$-Horizontes legt eine interglaziale Entstehung nahe. Nach der anschließenden Pseudovergleyung wurde der A-Horizont gekappt und eine weitere Lößlehmdecke sedimentiert. Stellt man beide Vorgänge in das Würmglazial, so ergibt sich für den liegenden Bodenkomplex ein eemzeitliches Alter. Die Kryoturbationen einschließlich der Sedimentation der unteren Lößlehmdecke fallen demnach in die ausgehende Rißvereisung, so daß die liegenden glazifluvialen Sedimente dem maximalen Rißvorstoß zuzuordnen sind. 


\subsection{Alter und Lage des Vergletscherungsrandes der maximalen Vereisung}

Nördlich der rißeiszeitlichen Vergletscherungsgrenze treten als Zeugen einer weiteren Vereisung vorwiegend Erratika aus paläozoischem Material auf (Abb. 6). Sie setzen unmittelbar östlich und westlich des rißeiszeitlichen Endmoränenbogens von Pouxeux ein. Der aus Buntsandstein bestehende Bois des Grandes Haies steigt auf über $600 \mathrm{~m}$ Höhe an. Hier befinden sich auf oder nahe den höchsten Kuppen kristalline Erratika weit oberhalb von $450 \mathrm{~m}$ Höhe, bis wohin der rißeiszeitliche Gletscherlobus gereicht hatte.

Nach $\mathrm{N}$ fällt das Gelände im Forêt Domaniale de Tanières auf $400-440 \mathrm{~m}$ ab, auf dem ebenfalls vereinzelte Granit- und Gneisgeschiebe erhalten sind. Weiter nördlich erhebt sich der Horst von Epinal, der am Rande des Moseldurchbruchs über $500 \mathrm{~m}$ ü. M. erreicht. FlaGEOLLET (1988: 20) nimmt an, daß die Eismassen der maximalen Vergletscherung die Kammlinie nicht mehr erreicht haben. Offensichtlich ist es jedoch im Bereich des schmalen Durchbruchstales zu einem Eisstau gekommen, denn auf der höchsten Erhebung, dem $515 \mathrm{~m}$ hohen Haut du Mont, befinden sich einzelne Erratika. Sie belegen für das $2 \mathrm{~km}$ entfernte Moseltal eine Eismächtigkeit von mehr als $100 \mathrm{~m}$. Das Eis hat demnach in breiter Front den Horst überschritten, ein Gletscherende im $5 \mathrm{~km}$ entfernten Stadtgebiet von Epinal ist daher durchaus wahrscheinlich. Morphologische Hinweise auf den damaligen Gletscherrand sind jedoch wegen der dichten Bebauung nicht mehr zu erkennen. Nach SERET (1966: 402f.) soll dort früher in ca. $350 \mathrm{~m}$ Höhe kurzfristig Moräne aufgeschlossen gewesen sein.

Nördlich des Horstes von Epinal fällt das Gelände deutlich unter $400 \mathrm{~m}$ ab, und die flachwellige Oberfläche wird von einer 370-380 m hohen Verebnung beherrscht. Westlich der Mosel setzt sie auf der Höhe von Epinal ein und erstreckt sich auf über $8 \mathrm{~km}$ im Rücken des Forêt Domaniale de Souche Thaon. Östlich der Mosel ist das Relief durch das Einschneiden des St. Oger stärker in einzelne Rücken aufgelöst, doch das 370-380 m-Niveau ist auch hier in Resten (z.B. Fort de Dogneville, Fort de Longchamp, zwischen Longchamp und Deyvillers) erhalten. FLAGEOLLET (1988: 20) interpretiert die in nur wenigen Anschnitten freigelegten Schotter als fluvioglaziale Ablagerungen, „die der ältesten Vereisung oder den ältesten Vereisungen" zuzuordnen sind. Gegen eine solche Deutung spricht sowohl die immense flächenhafte Ausdehnung als auch die konstante Höhenlage des Niveaus. Nach BONNEFONT (1986: 71f.) handelt es sich vielmehr aufgrund der intensiven Verwitterung und weiten Verbreitung der Schotter - sie treten u. a. in ähnlicher Höhenlage auch längs der Mortagne auf - um "piedmonts villafranchiens". Aus den morphologischen Gegebenheiten läßt sich ableiten, daß die 370-380 m-Fläche eine pliozäne bis altpleistozäne Moselterrasse ist, in die sich der Fluß bis zum maximalen Eisvorstoß bei Epinal auf ca. $320 \mathrm{~m}$ eingetieft hat.

Bei Golbey, $4 \mathrm{~km}$ nördlich des Stadtzentrums von Epinal, sind in ca. $335 \mathrm{~m}$ Höhe in einer Reihe von Kiesgruben glazifluviale Ablagerungen angeschnitten, die von SERET (1966: 439f.) als proglaziale Sedimente der maximalen Vergletscherung gedeutet werden. Nach FlaGEOLLET (1988: Abb. 2) handelt es sich um fluvioglaziale Schotter der Rißvereisung.

Im Sommer 1988 konnte bei Rang du Xay folgendes Profil (Abb. 5) aufgenommen werden: Die glazifluvialen Sedimente setzen sich aus einer wechselnden Folge von Geröll-, Kies- und Sandschichten in einer rötlichen Matrix zusammen. In diese Sedimente ist eine flache Delle eingeschnitten. Die glazifluvialen Sedimente schließen mit einem $40-50 \mathrm{~cm}$ mächtigen Auelehm ab, der intensiv verwittert ist. Einzelne Granit- und Gneisgerölle sind stark zersetzt. Der Schluffgehalt des $\mathrm{B}_{\mathrm{v}}$-Horizontes beträgt $73 \%$, der Tongehalt $18 \%$. Es folgt ein $30-40 \mathrm{~cm}$ mächtiger $B_{t}$ Horizont, dessen Basis in den liegenden Auelehmboden eingewürgt ist. Die hangenden Partien sind stark verdichtet und durch eine kräftige Pseudovergleyung überprägt worden. Den Abschluß bildet ein $50-60 \mathrm{~cm}$ mächtiger Löß, auf dem sich eine Braunerde entwickelt hat.

Interpretiert man sowohl den gekappten Auelehmboden als auch den folgenden kryoturbat eingewürgten $\mathrm{B}_{\mathrm{t}}$-Horizont mit der aufliegenden Pseudovergleyung als interglaziale Bodenbildungen, so scheint m. E. ein mindelzeitliches Alter der liegenden glazifluvialen Ablagerungen als höchstwahrscheinlich.

Cailuier (1977) hat in dem Boden, der den Schotterkomplex abschließt, Holzreste gefunden und aufgrund deren ${ }^{14} \mathrm{C}$-Alter von über 40.000 Jahren die Bodenbildung ins Brörup-Interstadial, später jedoch, aufgrund der intensiven Ausbildung, ins Eem gestellt (zit. nach FLAGEOLLET 1984: 185). Nimmt man allerdings für die Genese der Parabraunerde und der anschließenden Pseudovergleyung des hangenden Lößpaketes ebenfalls interglaziale Klimabedingungen an, so muß der liegende Schotterkomplex ins Mindelglazial gestellt werden, so daß sich die in Tab. 1 dargestellte Chronologie ergibt.

Vergleicht man die großflächig erhaltenen Relikte glazialer und glazifluvialer Formen und Sedimente der mittleren Vereisung mit den nur wenigen Vorkommen glazialer Sedimente und Erratika der ältesten Vereisung, so muß zwischen beiden Vergletsche- 


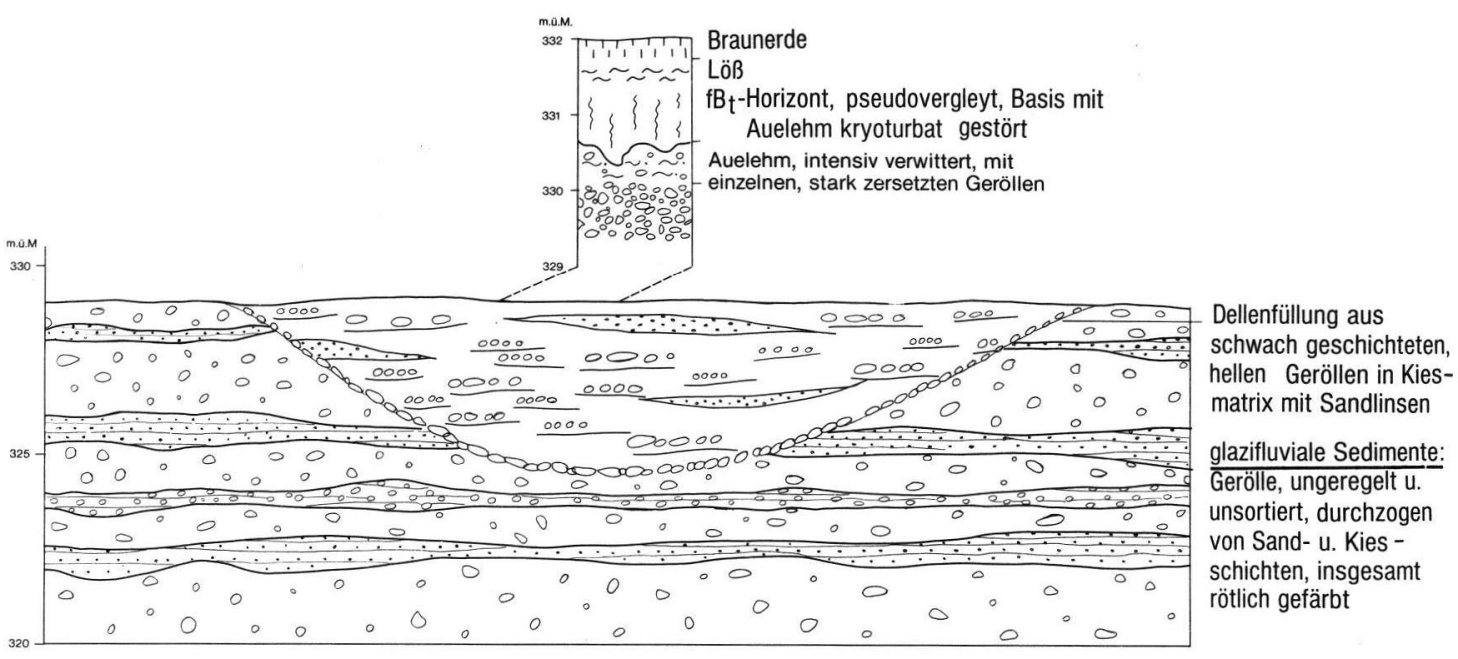

Abb. 5: Aufschluß Golbey: mindeleiszeitliche glazifluviale Sedimente und ihre Deckschichten.

Fig. 5: Coupe Golbey: sédiments glaciofluviaux du Mindelien et leurs couches de couverture.

Tab. 1: Zeitliche Gliederung der Morphodynamik und Bodenbildung im Aufschluß Golbey
Prozesse

Braunerdebildung

Lößablagerung

Kappung des Bodenkomplexes

Parabraunerdebildung

und Pseudovergleyung

Lößablagerung und Kryoturbation

Kappung des Auelehmbodens

Intensive Verwitterung

des Auelehms

Sedimentation des Auelehms

Rinnenbildung und Füllung

Ablagerung der glazifluvialen

Sedimente
A 1 t e r

rezent

Würm-Kaltzeit

Riß-Würm-

Interglazial

Riß-Kaltzeit

Mindel-Riß-

Interglazial

Mindel-Kaltzeit

\section{Glaziale und fluvioglaziale Formen und Ablagerungen im östlichen Untersuchungsgebiet}

\subsection{Die räumliche Ausdehnung der rißeiszeitlichen Vergletscherung}

Während für das Moseltal noch weitgehende Übereinstimmung der verschiedenen Bearbeiter in bezug auf die Lage der Endmoränen der ältesten und mittleren Vergletscherung besteht, gehen die Auffassungen über den jeweils maximalen Eisrand während der Mindel- und Rißvereisung im östlichen Untersuchungsgebiet weit auseinander. So reichen nach FLAGEOLLET (s. Abb. 3) östlich Docelles die rißeiszeitlichen Endmoränen fast bis an den mindeleiszeitlichen Gletscherrand heran, während nach SERET (s. Abb. 2) dieser Raum nur von der ältesten Vergletscherung erfaßt wurde und die mittlere Vereisung nicht über das Cleurietal hinauskam. Daher sollen zunächst für diesen Raum morphologische Kriterien zur Ermittlung der rißeiszeitlichen Vergletscherungsgrenze zusammengestellt werden.

rungen eine lange Zeit der Verwitterung und Abtragung geherrscht haben, d. h. die Zuordnung dieser Ablagerungen zu zwei verschiedenen Glazialen und nicht zu zwei Stadien einer Kaltzeit ist gerechtfertigt. Aufgrund der Zahl der interglazialen Bodenbildungen muß die älteste Vergletscherung mindestens ins Mindel, die mittlere mindestens ins Riß gestellt werden. Es ist davon auszugehen, daß auch die älteste Vergletscherung durch mehrere Vorstoßphasen gekennzeichnet war, diese sich jedoch aus den wenigen Relikten glazialer und fluvioglazialer Ablagerungen kaum noch rekonstruieren lassen.
Im Mündungsbereich des Barba in die Vologne lassen mehrere Aufschlüsse Aussagen über das Ausmaß der Vergletscherung in diesem Raum während der Rißvereisung zu (Abb. 6). Die Endmoräne des Barbagletschers befindet sich knapp $3 \mathrm{~km}$ vor der Mündung des Barba in die Vologne und zeigt eine Eismächtigkeit bis zur $440 \mathrm{~m}$-Isohypse an. Vorgelagert sind glazifluviale Sedimente, die teilweise auf der stark verfestigten mindeleiszeitlichen Grundmoräne liegen und bis in $418 \mathrm{~m}$ Höhe reichen. Die ca. $13 \mathrm{~m}$ mächtigen Ablagerungen sind schwach geschichtet und 


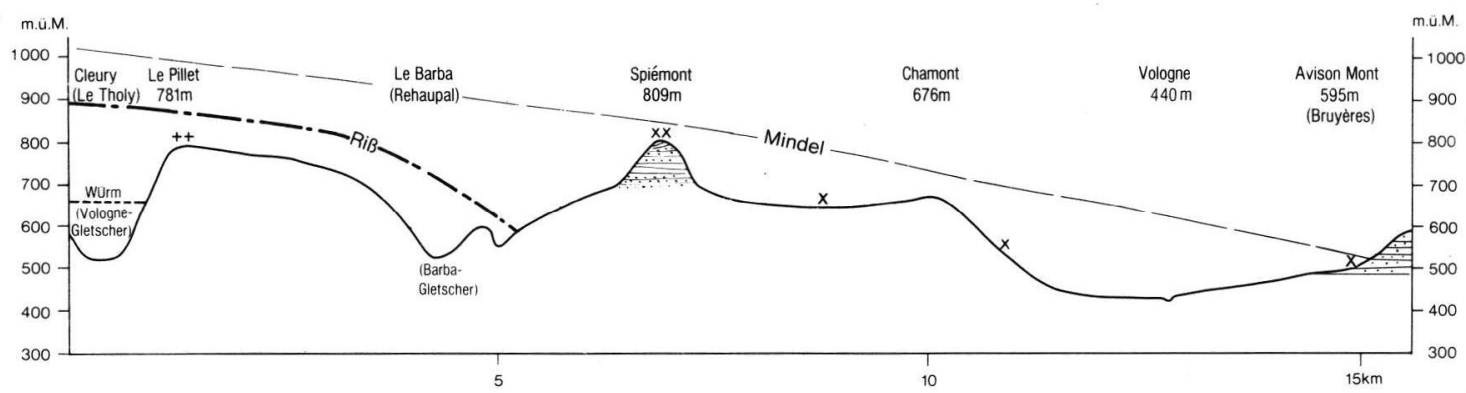

Abb. 6: Süd-Nord Höhenprofil zwischen Le Tholy und Bruyères mit Angabe der würm-, riß- und mindeleiszeitlichen Gletscherobergrenzen.

Fig. 6: Profil d'altitude du sud au nord entre Le Tholy et Bruyères avec hauteurs des glaciers du Würm, Riß et Mindel.

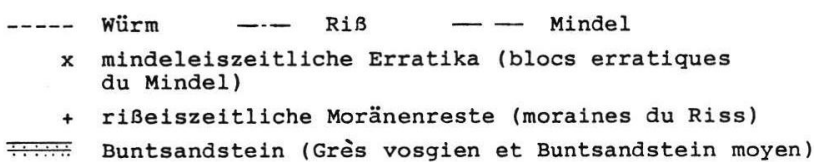

sortiert und zeichnen sich durch eine lagenweise auftretende Eisenverfärbung aus.

Im Volognetal fehlen entsprechende Aufschlüsse, um den weitesten rißeiszeitlichen Vorstoß zu bestimmen. Der Formenschatz selbst läßt eine eindeutige Ansprache kaum zu, so daß die Lage der Endmoräne nur durch vergleichende Schlüsse eingegrenzt werden kann. Auf jeden Fall ist das rißeiszeitliche Gletscherende aufgrund der Talweitung nördlich von Granges s. V. anzusetzen.

\subsection{Die Lage des Vergletscherungsrandes} während der Mindelvereisung — abgeleitet aus

Erratika-Funden und der rekonstruierten Eismächtigkeit

Über das Ausmaß der ältesten Vergletscherung im östlichen Untersuchungsgebiet (Abb. 1) bestehen bei den einzelnen Autoren erhebliche Meinungsunterschiede. Während HANTKE (1978: Karte 2) und RAHM (1970: Karte 1) den Gletscherrand bis nördlich von Bruyères reichen lassen, soll nach den Auffassungen von Flageollet (1984) der Eisrand 7 km, nach SERET (1966) sogar $12 \mathrm{~km}$ weiter südlich gelegen haben, d. h. der Raum Bruyères wäre demnach stets unvergletschert gewesen.

Im östlichen Untersuchungsgebiet ist die Funddichte außerhalb der rißeiszeitlichen Vergletscherung zwar relativ gering und die Längsachse der Kristallinblöcke beträgt oft weniger als einen Meter. Trotzdem ergeben sich aufgrund der Erratikaverbreitung aber Hinweise auf die Existenz mindestens einer weiteren Vereisung (Abb. 7).

Ein S-N-Profil (Abb. 7) mit der Lage der gesicherten Erratika zeigt, daß eine geschlossene Eiskappe den Raum bis Bruyères während der Mindeleiszeit überzogen hatte. Einen wichtigen Zeugen dieser Vergletscherung bildet der $809 \mathrm{~m}$ hohe aus Buntsandstein aufgebaute Inselberg Spiemont, der einem ca. $700 \mathrm{~m}$ hohen Rücken aufsitzt. Nach SERET (Abb. 1) befindet er sich außerhalb, nach FLAGEOLLET unmittelbar am Rand der maximalen Vergletscherung. Auf der Kuppe liegen mehrere bis zu 2,5 $\times 3,0 \mathrm{~m}$ große Findlinge aus verschiedenen Granitvarietäten. Es ergibt sich somit bei der Herkunft der Eismassen aus dem E bzw. SE eine Eismächtigkeit, die bezogen auf das nur $3 \mathrm{~km}$ entfernte Volognetal $300 \mathrm{~m}$ betragen haben muß. Der Gletscher im Volognetal war demnach ausreichend mächtig, um selbst bei deutlichem Abflachen über Bruyères hinaus bis an den ca. $500 \mathrm{~m}$ hohen Fuß des Horstes von Epinal vorzustoßen. Hiermit steht die niedrige Höhe der Wasserscheide zwischen Vologne und Mortagne im Einklang. Während sie sonst auf dem über $550 \mathrm{~m}$ hohen Bergrücken des Forêt de Faite verläuft, sind nördlich von Bruyères beide Flußsysteme nur durch flache Pässe verbunden. Es ist naheliegend, die Entwässerung dieses Raumes auf einen in ca. $500 \mathrm{~m}$ Höhe ausapernden Gletscherlobus zurückzuführen, dessen Schmelzwässer zum Teil nach $\mathrm{N}$ 


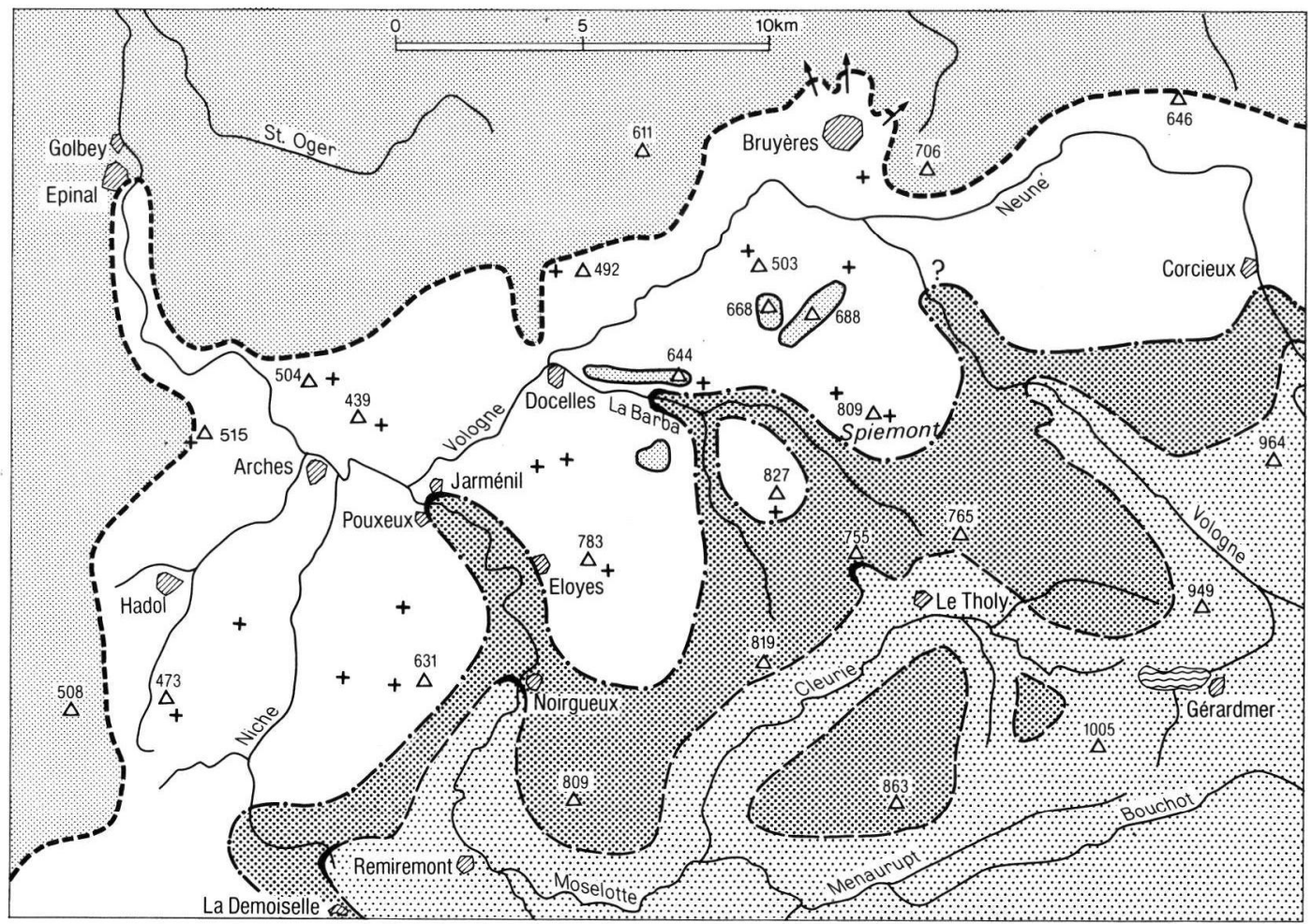

Entwurf : G.Wenzens

Kartographie : K.Massoud

Abb. 7: Ausdehnung der Vergletscherungen in den nördlichen Südvogesen.

Fig. 6: Extension des glaciations quaternaires dans la région des Vosges sud.

würm RiB
$\begin{aligned} & \text { stets unver- } \\ & \text { gletschert }\end{aligned}$
$+\begin{aligned} & \text { zone épargnée par } \\ & \text { mindeleiszeitliche Erratika/blocs erratiques du } \\ & \text { Mindel }\end{aligned}$
$\begin{aligned} & \text { Endmoränenbogen } \\ & \text { moraine frontale }\end{aligned}$
$\begin{aligned} & \text { Schmelzwasserabfluß bei } \\ & \text { Bruyères } \\ & \text { écoulement de l'eau de } \\ & \text { fusion à Bruyères }\end{aligned}$

abgegangen sind und dadurch die Wasserscheide auf das heutige Niveau erniedrigt haben. Auch ist eine pliozäne bis altquartäre S-N Entwässerung der heutigen Vologne zum Mortagne-System sehr wahrscheinlich (BONNEFONT (1986: 74). Das nahezu rechtwinklige Abbiegen des Vologne-Tales südlich von Bruyères könnte daher gut mit dem Abbiegen des mindeleiszeitlichen Gletschers in Verbindung stehen; der Unterlauf der Vologne wäre somit mindelzeitlichen Alters.

$\mathrm{Da}$ sich die höchsten Erratikavorkommen im Raum Bruyères in $500 \mathrm{~m}$ Höhe befinden, ergibt sich eine Eismächtigkeit von mindestens $70 \mathrm{~m}$. Die Eismassen sind von Bruyères aus dem Gefälle folgend nach SW abgeflossen. Aus den am weitesten nördlich vorkom- menden Granit- und Gneisgeschieben läßt sich von Bruyères bis Le Roulier ein Absinken der Gletscheroberfläche von über $500 \mathrm{~m}$ ü. M. auf unter $500 \mathrm{~m}$ ü. M. ableiten. So befindet sich $750 \mathrm{~m}$ nordwestlich von Le Roulier in $480 \mathrm{~m}$ Höhe ein $80 \mathrm{~cm}$ großer Granitblock, dessen Größe auf einen glazialen Transport schließen läßt. Dies würde bedeuten, daß der $515 \mathrm{~m}$ hohe N-S-verlaufende Rücken Bois de la Meule nördlich Docelles die Eismassen des Moselgletschers von denen des Volognegletschers getrennt hat. $\mathrm{Da}$ auch auf den über $600 \mathrm{~m}$ hohen Rücken Bois de Malenru (688 m. ü. M.), Steaumont (668 m ü. M.) und Bois de l'Encerf (644 m ü. M.) keine Erratika gefunden wurden, ist anzunehmen, daß sie als Nunatakker aus der sonst geschlossenen Eiskappe herausgeragt haben. 


\subsection{Eismächtigkeiten, Schneegrenzhöhen und Vergletscherungstypen in den nördlichen Südvogesen}

Im Gegensatz zum Südschwarzwald, der aufgrund seiner ausgedehnten Hochflächen in der Würm- und Rißkaltzeit von einer Kappenvereisung (RAHM 1970: Karte 2, SCHREINER 1985: 36) bedeckt war, deren Eismassen lediglich in den größeren Tälern zungenförmig vorstießen, geht man in den Vogesen wegen der intensiven Zerschneidung des gesamten Gebirgskörpers während der beiden jüngeren Vereisungsperioden von Talvergletscherungen aus. Dies trifft freilich nur für die Würmvereisung zu, wobei bezüglich der maximalen Vorstöße der einzelnen Talgletscher weitgehend Übereinstimmung herrscht. Über die Herkunft des Eises im unteren Cleurietal bestehen allerdings konträre Auffassungen. Nach SALOME (1968) und WENZENS (1987) vereinigten sich Vologne-, Mosel- und Moselottegletscher im ca. $400 \mathrm{~m}$ hohen Becken von Remiremont. Nach SERET (1966: 462f.) und FlaGEOLLET (1988: 22f.) wird der weiteste Volognevorstoß durch die Endmoränen westlich des Sees von Gérardmer bzw. durch den $4 \mathrm{~km}$ weiter westlich gelegenen Wall bei Bas Beillard markiert. Die glazialen Ablagerungen im unteren Cleurietal sollen nach beiden Autoren von einer Moselotte-Diffluenz- zunge stammen, deren Eismassen am Gletscherende bei Le Tholy bis zur 650 m-Isohypse reichten. Falls sich eine Diffluenzzunge tatsächlich $10 \mathrm{~km}$ talaufwärts bewegt hätte, müßte am Zusammentreffen von Mosel- und Moselottegletscher die Eisoberfläche mindestens bis in ca. $750 \mathrm{~m}$ Höhe gereicht haben, damit die gestauten Eismassen bis nach Le Tholy abfließen konnten. Nach SERET (1966: Fig. 22) kann jedoch die Gletscherhöhe in diesem Raum nur bei maximal $580 \mathrm{~m}$ ü. M. gelegen haben, da der $586 \mathrm{~m}$ hohe $\mathrm{Paß}$ Fontaine de Kake vom Eis nicht mehr überschritten wurde. Diese niedrige Lage bestätigt auch der sich nördlich erstreckende Endmoränenbogen von La Demoiselle in ca. $550 \mathrm{~m}$ Höhe sowie der nur in $6 \mathrm{~km}$ Entfernung bei Noirgueux stirnende Moselgletscher (WENZENS 1987: 86).

Die Rekonstruktion der rißeiszeitlichen Vergletscherungsgrenze (Abb. 6) zeigt im Gegensatz zur jüngeren Talvergletscherung bereits die charakteristischen Merkmale einer Kappenvereisung, wie sie auch in der Mindeleiszeit ausgebildet war. Aufgrund des wesentlich größeren Nährgebietes konnte sich eine Eiskappe bilden, aus der lediglich der $827 \mathrm{~m}$ hohe Inselberg l'Ormont herausragte und einen Gletscherlobus teilte. Auch im Mosel- und Volognetal bildeten sich Gletscherzungen.

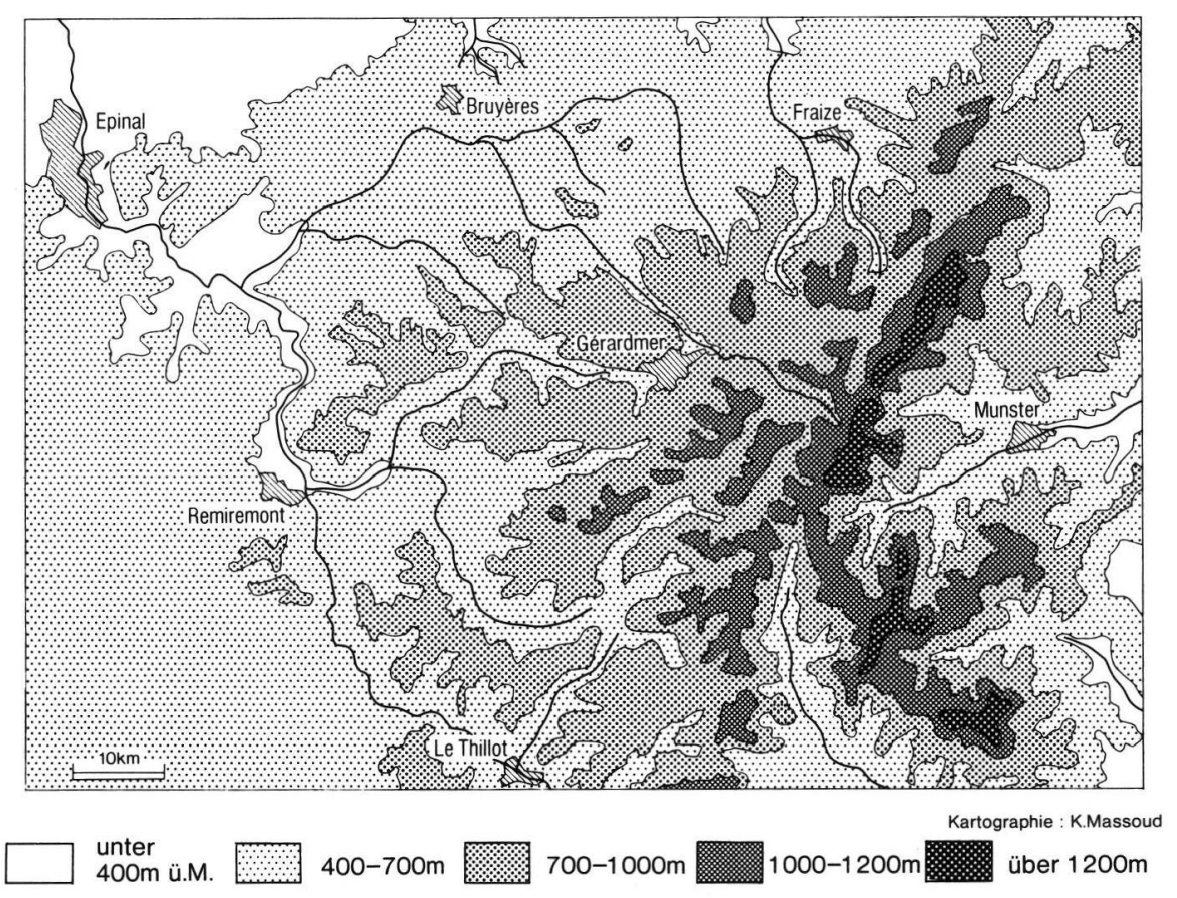

Abb. 8: Höhenschichtenkarte der Südvogesen.

Fig. 8: Carte des niveaux d'altitude des Vosges sud. 
In allen Arbeiten zur maximalen Vergletscherung der Vogesen wird eine Kappenvereisung angenommen, wobei nur in den größeren Tälern einzelne Loben weiter vorstießen. Die Karten von SERET (Abb. 2) und FLAGEOLLET (Abb. 3) zeigen innerhalb des Untersuchungsgebietes eine äußerst asymmetrische Flächenzunahme von der jüngsten zur ältesten Vereisung. Während im Moseltal die jeweiligen Gletscherenden (Noirgueux bzw. Epinal) $22 \mathrm{~km}$ voneinander entfernt sind, sind es im Volognetal nur $8 \mathrm{~km}$, wobei nach SERET an der rechten Talseite die Grenzen aller drei Vergletscherungsphasen sehr dicht beieinander liegen. Im Volognetal wäre demnach die Eismächtigkeit der mindelzeitlichen Kappenvereisung nur unwesentlich größer gewesen als die der würmeiszeitlichen Talvergletscherung.

Die vorgelegten Geländebefunde, die diesen Vorstellungen von SERET (1985) und FLAGEOLLET (1988) widersprechen, werden auch durch Berechnungen der Schneegrenzhöhen in den einzelnen Kaltzeiten bestätigt. Nach allgemeiner Auffassung lag die würmeiszeitliche Schneegrenze in den Südvogesen in ca. 850 m Höhe (DARMOIS 1973: 127; EGGERS 1964: 55; HANTKE 1978: 402). Überträgt man die Mindestabsenkung der rißeiszeitlichen Schneegrenze in den Alpen von $100-200 \mathrm{~m}$ gegenüber der würmeiszeitlichen auf die Südvogesen, so erhält man einen Wert von ca. $750 \mathrm{~m}$ (vgl. WeNZENS 1987: 90). Wegen der insgesamt wesentlich größeren Ausdehnung des Mindeleises ist für die maximale Vergletscherung ein Schneegrenzwert von mindestens $700 \mathrm{~m}$ anzusetzen. Nach den Berechnungen der Höhenschichtenareale von FREY (1965: 78) nimmt die Größe des Nährgebietes in den S-Vogesen bei Absenkung der Schneegrenze um jeweils $100 \mathrm{~m}$ überproportional zu:

\section{Höhenlage der Schneegrenze}

$900 \mathrm{~m}$ ü. $\mathrm{M}$.

$800 \mathrm{~m}$ ü. $\mathrm{M}$.

$700 \mathrm{~m}$ ü. $\mathrm{M}$.

\section{Größe des Nährgebietes}
$315 \mathrm{~km}^{2}$
$529 \mathrm{~km}^{2}$
$837 \mathrm{~km}^{2}$

Legt man nun eine Höhenschichtenkarte der Südvogesen $(A b b .8)$ zugrunde und berücksichtigt die über $700 \mathrm{~m}$ hohen Nährgebiete, so wird deutlich, daß die aufgrund von morphologischen Kriterien und der Rekonstruktion der Eismächtigkeit angegebene mindeleiszeitliche Vergletscherungsgrenze im Raum um Bruyères durch die Größe des Einzugsgebietes des Volognegletschers bestätigt wird. Der Moselgletscher konnte weiter vorstoßen, weil die Eiskappe im wesentlich tiefer eingeschnittenen Talboden - bei Remiremont liegt er bereits unter $400 \mathrm{~m}$ ü. M. - eine größere Mächtigkeit erreichte.

\section{Schriftenverzeichnis}

BONNEFONT, J. C. (1986): Les cailloutis anciens du piedmont occidental des Vosges et leur signification géomorphologique. - Revue Géogr. de l'Est, 26 (1-2): $71-80$; Nancy.

Caillier, M. (1977): Etude chronoséquentielle des sols sur terrasses alluviales de la Moselle. Genèse et évolution des sols lessivés glossiques. - Thèse doctorat spécialité. Univ. Nancy I, 87 S.; Nancy.

DARMOIS-THEOBALD, M. (1973): Recherches sur la morphologie glaciaire des vallées supérieures de la Meurthe (Vosges). - Annales Scientifiques de l'Université de Besançon 3 ème série, fasc. 21: 113-129; Besançon.

EGgERS, H. (1964): Schwarzwald und Vogesen. Ein vergleichender Überblick. - Westermann Taschenbuch Geographie 1: 144 S., 6 Kt., 5 Abb.; Braunschweig (Westermann).

FLAGEOLleT, J. C. (1984): Cent cinquante ans de recherches sur le glaciaire vosgien. Historique des idées et état de la question. - in Mélanges offerts au Professeur André Journaux: 173-192, 1 Kt.; Caen.

- (1988): Quartäre Vereisungen in den lothringischen Vogesen: Anzahl, Ausdehnung und Alter. - Eiszeitalter u. Gegenwart, 38: 17-36, 7 Abb.; Hannover.

FREY, C. (1965): Morphometrische Untersuchung der Vogesen. - Basler Beiträge zur Geogr. und Ethnol., Geogr. Reihe, 6: 150 S., 12 Abb., 6 Taf., 9 Beil.; Basel.

HANTKE, R. (1978): Eiszeitalter. Die jüngste Erdgeschichte der Schweiz und ihrer Nachbargebiete, 1: 398-411, 9 Phot., 2 Kt.; Thun (Ott).

Rahm, G. (1970): Die Vergletscherungen des Schwarzwaldes im Vergleich zu denjenigen der Vogesen. - Alem. Jb. 1966/67, 17: 257-272, 2 Kt.; Bühl-Baden.

SAlOME, A. I. (1968): A geomorphological study of the drainage area of the Moselotte and Upper Vologne in the Vosges (France). - Diss.: Univers. Utrecht; $98 \mathrm{~S}$., 17 Abb., 4 Tab., Diagr. u. Phot.; Utrecht.

SCHREINER, A. (1986): Neuere Untersuchungen zur Rißeiszeit im Wutachgebiet (Südostschwarzwald). - Jh. geol. Landesamt Baden-Württ., 28: 211-244, $10 \mathrm{Abb}$. 3 Tab.; Freiburg i. Br.

- \& MeTZ, B. (Hrsg.) (1985): Exkursionsführer I zur 22. wiss. Tagung in Freiburg i. Br., DEUQUA, $88 \mathrm{~S}$.; Hannover.

SERET, G. (1966): Les systèmes glaciaires du bassin de la Moselle et leurs enseignements. - Revue belge de Géographie, 90: 155-577, 77 Abb., 3 Tab., 15 Phot.; Brüssel.

- (1980): La stratigraphie des glaciations dans les vosges du sud. Problèmes de stratigraphie quaternaire en France et dans les pays limitrophes. - Suppl. Bull. A.F.E.Q., 1: 107-109, 1 Abb.; Lyon. 
SERET, G. (1985): Die eiszeitlichen Vergletscherungen der Lothringischen Vogesen und ihre Stratigraphie. DEUQUA-Exkursionsführer II: 15-97, 36 Abb., 3 Tab.; Hannover.

Service Géologique Nationale (1985): Carte géologique detaillée de la France au 1/50000, feuille Bruyères; Orléans.

VASKOU, P. (1984): La terrasse moyenne de la Moselle dans les Vosges. Essai de reconstitution de la genèse d'une terrasse fluviatile par rapport au cycle climatique quaternaire. - Bull. de l'Association francaise pour l'étude du Quaternaire, 1/2/3: 99-103; Paris.
WENZENS, G. (1987): Der kaltzeitliche Formenschatz im Cleurie-Tal (Südwestvogesen). - Eiszeitalter u. Gegenwart, 37: 79-91, 5 Abb.; Hannover.

Woillard, G. (1978): Grand Pile Peat Bog: A continous pollen record for the last 140,000 years. - Quaternary Research 9: 1-21, 9 Abb.; Washington.

Manuskript eingegangen am 20. 2. 1989, Nachträge Mai 1989. 